\title{
SYNTHESIS AND CHARACTERIZATION OF SCHIFF BASE SUBSTITUTED CARBAZOLE BEARING PYRIDOPYRIMIDINE COMPOUNDS
}

\author{
Arleta Rifati-Nixha ${ }^{1, *}$ and Mustafa Arslan ${ }^{2}$ \\ ${ }^{1}$ Chemistry Department, Faculty of Mathematical \& Natural Sciences, University of Prishtina, \\ Prishtina, 10000, Republic of Kosova \\ ${ }^{2}$ Chemistry Department, Faculty of Arts and Sciences, Sakarya University, \\ Sakarya, 54147, Turkey \\ *E-mail : arleta.rifati@uni-pr.edu
}

\begin{abstract}
The Schiff bases formation which is useful molecules both biologically and synthetically is one of the most important reactions in organic and medicinal chemistry. Available materials, catalyst-free and mild reaction conditions are essential to synthesize the Schiff bases in a good yield. In basic conditions, Schiff base substituted carbazole bearing pyridopyrimidine compounds (4a-n) were efficiently synthesized through corresponding benzaldehyde derivatives on treatment with 7- (4-amino-phenyl)-5-(9-ethyl-9H-carbazol-3-yl)- 1,3-dimethyl- 1Hpyrido [2,3-d] pyrimidine-2,4-dione in tetrahydrofuran under reflux in good yields. The structures of the synthesized compounds were identified by IR, ${ }^{1} \mathrm{H}$ NMR and ${ }^{13} \mathrm{C}$ NMR and elemental analysis.

Keywords: Carbazole, pyridopyrimidine, benzaldehyde, Schiff bases.
\end{abstract}

(C) RASĀYAN. All rights reserved

\section{INTRODUCTION}

Heterocyclic compounds, especially nitrogen containing, have been studied due to a variety of chemical and biological significance. In recent years, the pyrido-[2,3-d]-pyrimidine heterocyclic compounds that are annelated to a pyrimidine ring exhibit a wide range of biological and pharmacological properties ${ }^{1-3}$. The compounds show antitubercular ${ }^{4}$, calcium channel blockers $^{5}$ antibacterial $^{6}$, antiviral ${ }^{7}$, antifungal ${ }^{8.9}$, antimalarial ${ }^{10}$, antihypertensive ${ }^{11}$, carbonic anhydrase inhibitors ${ }^{12}$, analgesic and anti-inflammatory ${ }^{13,14}$ properties. Among the compounds, pyrimidine and pyridine-containing compounds, have been the subject of expanding research efforts in organic and biological chemistry. Interestingly, pyrimidine compounds are also known for possessing antitumor, anticancer and antineoplastic potencies ${ }^{15,16}$ and are very essential for synthetic drugs (e.g., barbituric acid derivatives), chemotherapeutic agents (e.g., sulfadiazine), and agricultural chemicals ${ }^{17}$.

The formation of carbon-nitrogen bonds are the most important transformations in organic chemistry ${ }^{18-24}$. Traditionally, imines are synthesized from the dehydrative condensation of amines and carbonyl compounds in the presence of an acid catalyst ${ }^{36,37}$ or by a number of catalytic and non-catalytic procedures, such as the self-condensation of amines upon oxidation ${ }^{38,39}$, the oxidation of secondary amines ${ }^{40,41}$ or directly from alcohols and amines via several tandem catalytic processes ${ }^{42,43,44}$, mostly with homogeneous systems. Imines, in particular, are very important as electrophilic reagents in many organic reactions, such as reductions, additions, condensations and cycloadditions ${ }^{25-35}$. This study investigated 14 new Schiff base substituted carbazole bearing pyridopyrimidine compounds.

\section{EXPERIMENTAL}

Material and Methods

A SHIMADZU Prestige-21 (200 VCE) spectrometer measured the IR spectra. The ${ }^{1} \mathrm{H}$ and ${ }^{13} \mathrm{C}$ chemical shifts referenced to the internal deuterated solvent. Respectively, the measurements of the ${ }^{1} \mathrm{H}$ and ${ }^{13} \mathrm{C}$

Rasayan J. Chem., 11(3), 1286-1293(2018)

http://dx.doi.org/10.31788/RJC.2018.1134014

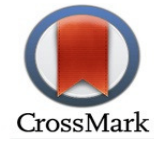


NMR spectra on spectrometer at VARIAN Infinity Plus 300 and at $75 \mathrm{~Hz}$. The elemental analysis was carried out with a Leco CHNS-932 instrument. Melting points were taken on a Yanagimoto micromelting point apparatus and are uncorrected. The preparation of the synthesis of carbazole containing pyridopyrimidine substituted Schiff base derivatives was completed in accordance with Scheme-1.
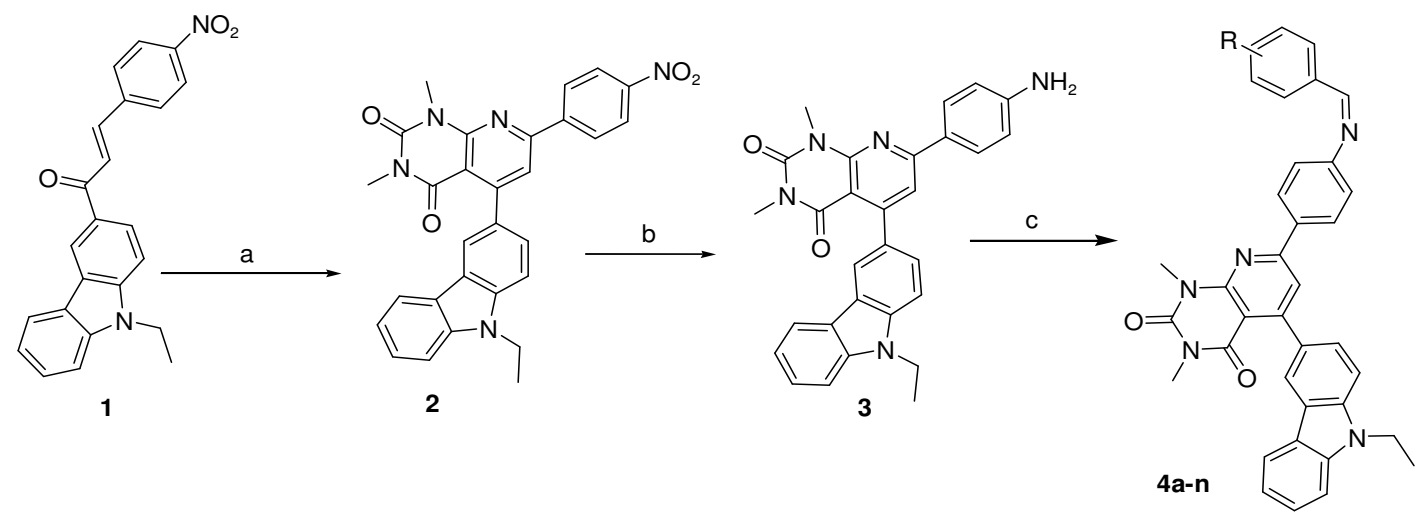

\begin{tabular}{c|c|c|c|c|c}
\hline Compounds & $\mathrm{R}$ & Compounds & $\mathrm{R}$ & Compounds & $\mathrm{R}$ \\
\hline $4 \mathrm{a}$ & $\mathrm{H}$ & $4 \mathrm{f}$ & $4-\mathrm{CH}_{3}$ & $4 \mathrm{k}$ & $2-\mathrm{NO}_{2}$ \\
\hline $4 \mathrm{~b}$ & $4-\mathrm{NO}_{2}$ & $4 \mathrm{~g}$ & $3-\mathrm{Cl}$ & $4 \mathrm{l}$ & 3,4 -dichlor \\
\hline $4 \mathrm{c}$ & $4-\mathrm{F}$ & $4 \mathrm{~h}$ & $3-\mathrm{Br}$ & $4 \mathrm{~m}$ & $2-\mathrm{Br}$ \\
\hline $4 \mathrm{~d}$ & $4-\mathrm{OCH}_{3}$ & $4 \mathrm{i}$ & $3,4-$ dimethoxy & $4 \mathrm{n}$ & 2,5 -dimethoxy \\
\hline $4 \mathrm{e}$ & $3-\mathrm{OCH}_{3}$ & $4 \mathrm{j}$ & $3-\mathrm{CH}_{3}$ & & \\
\hline
\end{tabular}

Scheme-1: Synthetic route of Schiff Base Substituted Carbazole containing Pyrido-Pyrimidine Compounds. Reagents and Yields: (a) 6-Amino-1,3-Dimethyluracil, $\mathrm{NaOH}$, EtOH, Reflux, 7 h, 90\%; (b) $\mathrm{NiCl}_{2}, \mathrm{NaBH}_{4}$, THF, $0{ }^{\circ} \mathrm{C}, 8 \mathrm{~h}, 78 \%$; (c) Aldehyde, $\mathrm{Na}_{2} \mathrm{CO}_{3}$, THF, $70^{\circ} \mathrm{C}, 24 \mathrm{~h}, 72-88 \%$.

\section{Synthesis of Carbazole Substituted Pyridopyrimidine}

A solution of nitrochalcone (was prepared according to the following procedures mentioned in literature $\left.{ }^{45}\right)(2.16 \mathrm{~g}, 5.84 \mathrm{mmol})$ in a mixture of ethanol $(50 \mathrm{ml}), 6$-amino-1,3-dimethyluracil $(0.89 \mathrm{~g}, 5.74 \mathrm{mmol})$ and sodium hydroxide $(0.22 \mathrm{~g}, 5.5 \mathrm{mmol})$ was heated at reflux for 7 hours, quenched with water, filtered and recrystallized from diethyl ether.

\section{General Procedure for the Synthesis of Amino Pyridopyrimidino Derivatives}

$\mathrm{NaBH}_{4}(1.23 \mathrm{~g}, 32.37 \mathrm{mmol})$ was added portionwise at $0{ }^{\circ} \mathrm{C}$ (ice bath) over $10 \mathrm{~min}$ to a solution of 5-(9Ethyl-9H-carbazol-3-yl)-1,3-dimethyl-7-(4-nitro-phenyl)- 1H-pyrido [2,3-d] pyrimidine -2,4-dione (2) $(1.5 \mathrm{~g}, 2.96 \mathrm{mmol})$ in anhydrous tetrahydrofuran and $\mathrm{NiCl}_{2}(3.11 \mathrm{~g}, 23.92 \mathrm{mmol})$. Stirring was continuing for $30 \mathrm{~min}$ with cooling, followed by stirring at room temperature for $2 \mathrm{~h}$. The mixture was evaporated off after the disappearing of the starting material. The black precipitate was dissolved in $1 \mathrm{~N} \mathrm{HCl}$ and then the acidic solution was alkalified by the addition of $1 \mathrm{~N} \mathrm{NaOH}$. After completion of the reaction, it was extracted with ethyl acetate $(20 \mathrm{ml})$ and washed with water and brine. The organic layer was dried over $\mathrm{MgSO}_{4}$ and concentrated (rotary evaporator) to give the crude products.

\section{General Procedure for Synthesis of Carbazole containing Pyridopyrimidino Substituted Imine} Derivatives (4a-n)

A mixture of 7- (4-Amino-phenyl)-5- (9-ethyl-9H-carbazol-3-yl)- 1,3-dimethyl- 1H-pyrido [2,3-d] pyrimidine-2,4-dione $1(0.8 \mathrm{~g}, 1.677 \mathrm{mmol})$ and benzaldehyde derivatives $(0.6 \mathrm{ml}, 5.143 \mathrm{mmol})$ in anhydrous tetrahidrofuran in presence of $\mathrm{Na}_{2} \mathrm{CO}_{3}(0.71 \mathrm{~g}, 6.454 \mathrm{mmol})$ was stirred at $70{ }^{\circ} \mathrm{C}$ for 24 hours. The solution was extracted with AcOEt with an aqoues phase. Organic phase was washed three times with water, dried with anhydrous magnesium sulphate and evaporated under reduced pressure. 


\section{RASĀYAN J. Chem.}

Vol. 11 | No. 3 |1286 - 1293 | July - September | 2018

7-[4-(Benzylidene- amino)- phenyl]- 5 -(9-ethyl-9H-carbazol-3-yl)-1,3-dimethyl- 1H- pyrido [2,3-d]pyrimidine-2,4-dione (4a)

It was obtained after recrystallization from diethylether to give as yellow crystals. Yield $76 \%$, m.p. $241.6{ }^{\circ} \mathrm{C}$; IR $\left(\mathrm{KBr}, v, \mathrm{~cm}^{-1}\right): 1660.7\left(\mathrm{R}_{2} \mathrm{C}=\mathrm{N}-\mathrm{R}\right), 2956.9(\mathrm{C}=\mathrm{C}$, aromatic $), 1703.1(\mathrm{C}=\mathrm{O}) ;{ }^{1} \mathrm{HNMR}(300$ $\left.\mathrm{MHz}, \mathrm{CDCl}_{3}, \delta, \mathrm{ppm}\right): 8.89(1 \mathrm{H}, \mathrm{s},=\mathrm{CH}), 8.59(1 \mathrm{H}, \mathrm{s},=\mathrm{CH}), 8.35-8.38(1 \mathrm{H}, \mathrm{d},=\mathrm{CH}), 8.19-8.22(1 \mathrm{H}$, $\mathrm{d},=\mathrm{CH}), 7.94-7.97(1 \mathrm{H}, \mathrm{t},=\mathrm{CH}), 7.64(1 \mathrm{H}, \mathrm{s},=\mathrm{CH}), 7.27-7.53(12 \mathrm{H}, \mathrm{m},-\mathrm{Ar}-\mathrm{H}), 4.39-4.47(2 \mathrm{H}, \mathrm{q}$, - $\left.\mathrm{CH}_{2}\right), 3.97\left(3 \mathrm{H}, \mathrm{s},-\mathrm{CH}_{3}\right), 3.43\left(3 \mathrm{H}, \mathrm{s},-\mathrm{CH}_{3}\right), 1.47-1.51\left(3 \mathrm{H}, \mathrm{t},-\mathrm{CH}_{3}\right) .{ }^{13} \mathrm{CNMR}\left(300 \mathrm{MHz}, \mathrm{CDCl}_{3}, \delta\right.$, ppm) : 14.1, 28.7, 30.5, 37.7, 109.5, 110.1, 110.9, 111.1, 118.2, 119.8, 119.9, 120.2, 120.3, 120.7, 123.3 (2C), 123.7, 125.5, 125.6, 125.7, 128.1, 128.3, 128.9, 129.0, 131.7, 134.6, 136.4, 137.7, 137.9, 150.4, 151.9, 152.0, 157.6, 159.9, 160.8, 163.9. Anal. Calcd. for $\mathrm{C}_{36} \mathrm{H}_{29} \mathrm{~N}_{5} \mathrm{O}_{2}: \mathrm{C}, 76.71 ; \mathrm{H}, 5.19 ; \mathrm{N}, 12.43$. Found: C, 76.03; H, 4.88; N, 11.96 .

5-(9- Ethyl- 9H- carbazol-3-yl)- 1,3-dimethyl-7- \{4-[(4-nitro-benzylidene)-amino]- phenyl\}-1Hpyrido[2,3-d]-pyrimidine-2,4-dione (4b)

It was recrystallized from diethylether to give as orange crystals. Yield $75 \%$, m.p. $234.6^{\circ} \mathrm{C} ; \mathrm{IR}(\mathrm{KBr}, v$, $\left.\mathrm{cm}^{-1}\right)$ : $1654.9\left(\mathrm{R}_{2} \mathrm{C}=\mathrm{N}-\mathrm{R}\right), 3053.3\left(\mathrm{C}=\mathrm{C}\right.$, aromatic), $1701.2(\mathrm{C}=\mathrm{O}), 1583.6\left(\mathrm{NO}_{2}\right) ;{ }^{1} \mathrm{HNMR}(300 \mathrm{MHz}$, $\left.\mathrm{CDCl}_{3}, \delta, \mathrm{ppm}\right): 8.89(1 \mathrm{H}, \mathrm{s},=\mathrm{CH}), 8.67(1 \mathrm{H}, \mathrm{s},=\mathrm{CH}), 8.34-8.37(1 \mathrm{H}, \mathrm{d},=\mathrm{CH}), 8.11-8.14(1 \mathrm{H}, \mathrm{d}$, $=\mathrm{CH}), 7.64(1 \mathrm{H}, \mathrm{s},=\mathrm{CH}), 7.27-7.54(12 \mathrm{H}, \mathrm{m},-\mathrm{Ar}-\mathrm{H}), 4.40-4.45\left(2 \mathrm{H}, \mathrm{q},-\mathrm{CH}_{2}\right), 3.97\left(3 \mathrm{H}, \mathrm{s},-\mathrm{CH}_{3}\right), 3.43$ $\left(3 \mathrm{H}, \mathrm{s},-\mathrm{CH}_{3}\right), 1.47-1.52\left(3 \mathrm{H}, \mathrm{t},-\mathrm{CH}_{3}\right) .{ }^{13} \mathrm{CNMR}\left(300 \mathrm{MHz}, \mathrm{CDCl}_{3}, \delta, \mathrm{ppm}\right): 14.2,28.7,30.4,38.3$, $105.6,108.6,109.9,111.0,112.9,114.4,118.9,119.0,119.9,120.4,120.6,123.3,123.4,124.1,124.2$, 124.5, 125.8, 125.9, 127.6, 130.9, 131.5, 137.4, 137.7, 138.1, 139.8, 149.1, 150.7, 151.9, 154.4, 159.0, 160.3, 161.3. Anal. Calcd. for $\mathrm{C}_{36} \mathrm{H}_{28} \mathrm{~N}_{6} \mathrm{O}_{4}$ : C, 71.04; H, 4.64; N, 13.81.Found: $\mathrm{C}$, 70.96; H, 4.57; N, 13.74 .

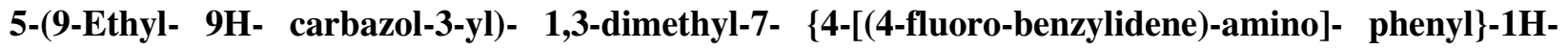
pyrido[2,3-d]-pyrimidine-2,4-dione (4c)

It was obtained after recrystallization from diethylether to give as yellow crystals. Yield 77.9\%, m.p. $256.5^{\circ} \mathrm{C}$; IR ( $\left.\mathrm{KBr}, v, \mathrm{~cm}^{-1}\right): 1660.7\left(\mathrm{R}_{2} \mathrm{C}=\mathrm{N}-\mathrm{R}\right), 3051.4(\mathrm{C}=\mathrm{C}$, aromatic), $1703.1(\mathrm{C}=\mathrm{O}), 1145.7(\mathrm{C}-\mathrm{F})$; ${ }^{1} \mathrm{HNMR}\left(300 \mathrm{MHz}, \mathrm{CDCl}_{3}, \delta, \mathrm{ppm}\right): 8.89(1 \mathrm{H}, \mathrm{s},=\mathrm{CH}), 8.55(1 \mathrm{H}, \mathrm{s},=\mathrm{CH}), 8.34-8.37(1 \mathrm{H}, \mathrm{d},=\mathrm{CH})$, 8.19-8.21 $(1 \mathrm{H}, \mathrm{d},=\mathrm{CH}), 7.93-7.96(1 \mathrm{H}, \mathrm{t},=\mathrm{CH}), 7.63(1 \mathrm{H}, \mathrm{s},=\mathrm{CH}), 7.16-7.53(11 \mathrm{H}, \mathrm{m},-\mathrm{Ar}-\mathrm{H}), 4.39-$ $4.44\left(2 \mathrm{H}, \mathrm{q},-\mathrm{CH}_{2}\right), 3.97\left(3 \mathrm{H}, \mathrm{s},-\mathrm{CH}_{3}\right), 3.42\left(3 \mathrm{H}, \mathrm{s},-\mathrm{CH}_{3}\right), 1.46-1.51\left(3 \mathrm{H}, \mathrm{t},-\mathrm{CH}_{3}\right) .{ }^{13} \mathrm{CNMR}(300$ $\mathrm{MHz}, \mathrm{CDCl}_{3}, \delta$, ppm) : 14.5, 28.7, 30.5, 37.9, 109.2, 110.0, 110.1, 110.2, 116.5, 116.8, 117.9, 118.6, $120.0,120.1,120.8,120.9,122.9,123.2,123.3,125.6,126.1,126.9,130.2,130.9,131.7,131.8,132.5$, 133.2, 138.1, 138.9, 151.5, 151.6, 157.1, 159.5, 160.2, 161.0, 162.3. Anal. Calcd. for $\mathrm{C}_{36} \mathrm{H}_{28} \mathrm{FN}_{5} \mathrm{O}_{2}: \mathrm{C}$, 74.34; H, 4.85; N, 12.04. Found: C, 74.26; H, 4.80; N, 12.01 .

5-(9-Ethyl-9H-carbazol-3-yl)- 1,3-dimethyl-7-\{4-[(4-methoxy-benzylidene)-amino]-phenyl\}-1Hpyrido [2,3-d]-pyrimidine-2,4-dione (4d)

It was recrystallized from ether to give as yellow crystals. Yield $72.3 \%$, m.p. $257.3^{\circ} \mathrm{C}$; IR $\left(\mathrm{KBr}, \mathrm{v}, \mathrm{cm}^{-1}\right)$ : $1660.7\left(\mathrm{R}_{2} \mathrm{C}=\mathrm{N}-\mathrm{R}\right), 2972.6\left(\mathrm{C}=\mathrm{C}\right.$, aromatic), $1703.1(\mathrm{C}=\mathrm{O}), 2839.9\left(\mathrm{O}_{-} \mathrm{CH}_{3}\right) ;{ }^{1} \mathrm{HNMR}(300 \mathrm{MHz}$, $\left.\mathrm{CDCl}_{3}, \delta, \mathrm{ppm}\right): 8.85(1 \mathrm{H}, \mathrm{s},=\mathrm{CH}), 8.49(1 \mathrm{H}, \mathrm{s},=\mathrm{CH}), 8.32-8.35(1 \mathrm{H}, \mathrm{d},=\mathrm{CH}), 8.17-8.19(1 \mathrm{H}, \mathrm{d}$, $=\mathrm{CH}), 7.87-7.89(1 \mathrm{H}, \mathrm{t},=\mathrm{CH}), 7.59(1 \mathrm{H}, \mathrm{s},=\mathrm{CH}), 7.23-7.51(11 \mathrm{H}, \mathrm{m},-\mathrm{Ar}-\mathrm{H}), 4.35-4.41\left(2 \mathrm{H}, \mathrm{q},-\mathrm{CH}_{2}\right)$, $3.92\left(3 \mathrm{H}, \mathrm{s},-\mathrm{OCH}_{3}\right), 3.88\left(3 \mathrm{H}, \mathrm{s},-\mathrm{CH}_{3}\right), 3.39\left(3 \mathrm{H}, \mathrm{s},-\mathrm{CH}_{3}\right), 1.44-1.49\left(3 \mathrm{H}, \mathrm{t},-\mathrm{CH}_{3}\right) .{ }^{13} \mathrm{CNMR}(300$ $\left.\mathrm{MHz}, \mathrm{CDCl}_{3}, \delta, \mathrm{ppm}\right): 14.1,28.7,30.5,38.0,55.7,105.7,108.9,109.2,114.4,114.5,118.2,118.3$, $119.8,119.9,120.2,120.3,120.7,120.8,120.9,123.3,123.5,123.6,125.6,125.7,126.4,126.5,128.2$, 129.3, 129.5, 130.9, 151.9, 152.428, 153.546, 154.665, 160.3, 160.9, 162.5. Anal. Calcd. for $\mathrm{C}_{37} \mathrm{H}_{31} \mathrm{~N}_{5} \mathrm{O}_{3}$ : C, 74.86; H, 5.26; N, 11.80. Found : C, 74.82; H, 5.19; N, 11.74.

5-(9-Ethyl-9H-carbazol-3-yl)- 1,3-dimethyl-7-\{4-[(3-methoxy-benzylidene)-amino]-phenyl\}-1Hpyrido[2,3-d]-pyrimidine-2,4-dione (4e)

It was obtained after recrystallization from diethylether to give as yellow crystals. Yield $75 \%$ m.p. $225.7^{\circ} \mathrm{C}$; IR $\left(\mathrm{KBr}, v, \mathrm{~cm}^{-1}\right)$ : $1658.8\left(\mathrm{R}_{2} \mathrm{C}=\mathrm{N}-\mathrm{R}\right), 2966.5(\mathrm{C}=\mathrm{C}$, aromatic), $1701.2(\mathrm{C}=\mathrm{O})$, $2933.7(\mathrm{O}-$ 
$\left.\mathrm{CH}_{3}\right) ;{ }^{1} \mathrm{HNMR}\left(300 \mathrm{MHz}, \mathrm{CDCl}_{3}, \delta, \mathrm{ppm}\right): 8.89(1 \mathrm{H}, \mathrm{s},=\mathrm{CH}), 8.56(1 \mathrm{H}, \mathrm{s},=\mathrm{CH}), 8.34-8.38(1 \mathrm{H}, \mathrm{d}$, $=\mathrm{CH}), 8.19-8.22(1 \mathrm{H}, \mathrm{d},=\mathrm{CH}), 7.63(1 \mathrm{H}, \mathrm{s},=\mathrm{CH}), 7.26-7.57(12 \mathrm{H}, \mathrm{m},-\mathrm{Ar}-\mathrm{H}), 4.39-4.47\left(2 \mathrm{H}, \mathrm{q},-\mathrm{CH}_{2}\right)$, $3.97\left(3 \mathrm{H}, \mathrm{s},-\mathrm{OCH}_{3}\right), 3.92\left(3 \mathrm{H}, \mathrm{s},-\mathrm{CH}_{3}\right), 3.43\left(3 \mathrm{H}, \mathrm{s},-\mathrm{CH}_{3}\right), 1.47-1.51\left(3 \mathrm{H}, \mathrm{t},-\mathrm{CH}_{3}\right) .{ }^{13} \mathrm{CNMR}(300$ $\mathrm{MHz}, \mathrm{CDCl}_{3}, \delta$, ppm): 14.1, 28.7, 30.4, 38.0, 55.7, 105.7, 109.1, 109.2, 112.2, 114.5, 118.6, 119.8, 119.9, $120.2,120.3,120.8,120.9,121.8,122.7,123.6,123.7,125.6,125.7,126.5,126.6,128.5,129.8,130.1$, 137.9, 138.0, 151.9 (2C), 152.0, 155.3, 160.9, 161.0, 161.1. Anal. Calcd. for $\mathrm{C}_{37} \mathrm{H}_{31} \mathrm{~N}_{5} \mathrm{O}_{3}: \mathrm{C}, 74.86 ; \mathrm{H}$, 5.26; N, 11.80. Found: C, 74.78; H, 5.21; N, 11.73 .

5-(9-Ethyl-9H-carbazol-3-yl)- $\quad$ 1,3-dimethyl-7- $\quad\{4-[(4-m e t h y l-b e n z y l i d e n e)-a m i n o]-\quad$ phenyl $\}-1 H-$ pyrido[2,3-d]-pyrimidine-2,4-dione (4f)

It was recrystallized from diethylether to give as orange crystals. Yield $73.5 \%$ m.p. $276.4^{\circ} \mathrm{C}$; $\mathrm{IR}(\mathrm{KBr}$, v, $\left.\mathrm{cm}^{-1}\right): 1658.8\left(\mathrm{R}_{2} \mathrm{C}=\mathrm{N}-\mathrm{R}\right), 3049.5(\mathrm{C}=\mathrm{C}$, aromatic $), 1703.1 \quad(\mathrm{C}=\mathrm{O}), 2951.1\left(-\mathrm{CH}_{3}\right) ;{ }^{1} \mathrm{HNMR}(300$ $\left.\mathrm{MHz}, \mathrm{CDCl}_{3}, \delta, \mathrm{ppm}\right): 8.89(1 \mathrm{H}, \mathrm{s},=\mathrm{CH}), 8.55(1 \mathrm{H}, \mathrm{s},=\mathrm{CH}), 8.35-8.38(1 \mathrm{H}, \mathrm{d},=\mathrm{CH}), 8.19-8.22(1 \mathrm{H}$, $\mathrm{d},=\mathrm{CH}), 7.64(1 \mathrm{H}, \mathrm{s},=\mathrm{CH}), 7.26-7.85(12 \mathrm{H}, \mathrm{m},-\mathrm{Ar}-\mathrm{H}), 4.41-4.44\left(2 \mathrm{H}, \mathrm{q},-\mathrm{CH}_{2}\right), 3.97\left(3 \mathrm{H}, \mathrm{s},-\mathrm{CH}_{3}\right)$, $3.43\left(3 \mathrm{H}, \mathrm{s},-\mathrm{CH}_{3}\right), 2.44\left(3 \mathrm{H}, \mathrm{s},-\mathrm{CH}_{3}\right), 1.46-1.51\left(3 \mathrm{H}, \mathrm{t},-\mathrm{CH}_{3}\right) \cdot{ }^{13} \mathrm{CNMR}\left(300 \mathrm{MHz}, \mathrm{CDCl}_{3}, \delta, \mathrm{ppm}\right)$ : $14.1,21.9,28.7,30.5,38.1,105.7,109.0,109.2$, 118.2, 119.9, 120.3, 120.7, 120.9, 123.3, 123.7, 125.7, $126.5,126.6,128.3,129.2,129.3,129.4,129.8,129.9,133.9,134.9,137.5,140.8,141.8,142.3,151.9$, 152.1, 152.3, 154.7, 160.3, 161.0, 161.1. Anal. Calcd. for $\mathrm{C}_{37} \mathrm{H}_{31} \mathrm{~N}_{5} \mathrm{O}_{2}: \mathrm{C}, 76.93 ; \mathrm{H}, 5.41 ; \mathrm{N}, 12.12$. Found: C, 76.85; H, 5.38; N, 12.04 .

5-(9- Ethyl- 9H- carbazol-3-yl)- 1,3-dimethyl-7-\{4-[(3-chlor-benzylidene)-amino]- phenyl\}-1Hpyrido[2,3-d]-pyrimidine-2,4-dione (4g)

It was obtained after recrystallization from diethylether to give as yellow crystals. Yield $88.2 \%$, m.p. $234.7^{\circ} \mathrm{C}$; IR $\left(\mathrm{KBr}, v, \mathrm{~cm}^{-1}\right): 1658.8\left(\mathrm{R}_{2} \mathrm{C}=\mathrm{N}-\mathrm{R}\right), 3061.0(\mathrm{C}=\mathrm{C}$, aromatic $), 1701.2(\mathrm{C}=\mathrm{O}), 746.5(\mathrm{C}-$ $\mathrm{Cl}) ;{ }^{1} \mathrm{HNMR}\left(300 \mathrm{MHz}, \mathrm{CDCl}_{3}, \delta, \mathrm{ppm}\right): 8.89(1 \mathrm{H}, \mathrm{s},=\mathrm{CH}), 8.54(1 \mathrm{H}, \mathrm{s},=\mathrm{CH}), 8.35-8.38(1 \mathrm{H}, \mathrm{d}$, $=\mathrm{CH}), 8.19-8.22(1 \mathrm{H}, \mathrm{d},=\mathrm{CH}), 7.63(1 \mathrm{H}, \mathrm{s},=\mathrm{CH}), 7.26-7.99(12 \mathrm{H}, \mathrm{m},-\mathrm{Ar}-\mathrm{H}), 4.39-4.45\left(2 \mathrm{H}, \mathrm{q},-\mathrm{CH}_{2}\right)$, $3.97\left(3 \mathrm{H}, \mathrm{s},-\mathrm{CH}_{3}\right), 3.43\left(3 \mathrm{H}, \mathrm{s},-\mathrm{CH}_{3}\right), 1.47-1.51\left(3 \mathrm{H}, \mathrm{t},-\mathrm{CH}_{3}\right) .{ }^{13} \mathrm{CNMR}\left(300 \mathrm{MHz}, \mathrm{CDCl}_{3}, \delta, \mathrm{ppm}\right)$ : $14.1,28.7,30.5,38.1,108.9,109.2,109.3,118.1,119.9,120.3,120.7,120.9,121.0,123.3$ (2C), 123.7, $125.7,126.6,126.7,127.5,128.2,128.6,129.3,129.8,130.3,133.2,135.2,138.2,140.8,141.8,151.5$, $151.9,152.1,160.3,160.4,161.0$. Anal. Calcd. for $\mathrm{C}_{36} \mathrm{H}_{28} \mathrm{ClN}_{5} \mathrm{O}_{2}: \mathrm{C}, 72.29 ; \mathrm{H}, 4.72 ; \mathrm{N}, 11.71$. Found: C, $72.05 ; \mathrm{H}, 4.63 ; \mathrm{N}, 11.59$.

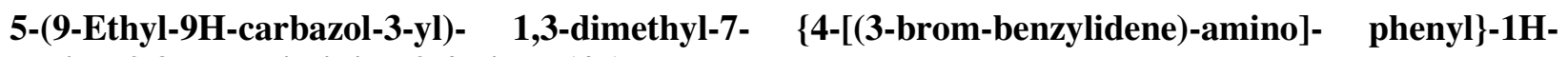
pyrido[2,3-d]-pyrimidine-2,4-dione (4h)

It was recrystallized from diethylether to give as yellow crystals. Yield $85.2 \%$, m.p. $233.2^{\circ} \mathrm{C}$; $\mathrm{IR}(\mathrm{KBr}$, v, $\left.\mathrm{cm}^{-1}\right): 1658.8\left(\mathrm{R}_{2} \mathrm{C}=\mathrm{N}-\mathrm{R}\right), 3057.2(\mathrm{C}=\mathrm{C}$, aromatic $), 1703.1 \quad(\mathrm{C}=\mathrm{O}), 678.9(\mathrm{C}-\mathrm{Br}) ;{ }^{1} \mathrm{HNMR}(300$ $\left.\mathrm{MHz}, \mathrm{CDCl}_{3}, \delta, \mathrm{ppm}\right): 8.88(1 \mathrm{H}, \mathrm{s},=\mathrm{CH}), 8.52(1 \mathrm{H}, \mathrm{s},=\mathrm{CH}), 8.34-8.38(1 \mathrm{H}, \mathrm{d},=\mathrm{CH}), 8.19-8.22(1 \mathrm{H}$, $\mathrm{d},=\mathrm{CH}), 8.14(1 \mathrm{H}, \mathrm{s},=\mathrm{CH}), 7.63(1 \mathrm{H}, \mathrm{s},=\mathrm{CH}), 7.27-7.53(11 \mathrm{H}, \mathrm{m},-\mathrm{Ar}-\mathrm{H}), 4.39-4.46\left(2 \mathrm{H}, \mathrm{q},-\mathrm{CH}_{2}\right)$, $3.96\left(3 \mathrm{H}, \mathrm{s},-\mathrm{CH}_{3}\right), 3.42\left(3 \mathrm{H}, \mathrm{s},-\mathrm{CH}_{3}\right), 1.46-1.51\left(3 \mathrm{H}, \mathrm{t},-\mathrm{CH}_{3}\right) \cdot{ }^{13} \mathrm{CNMR}\left(300 \mathrm{MHz}, \mathrm{CDCl}_{3}, \delta, \mathrm{ppm}\right)$ : $14.1,28.7,30.5,38.1,105.7,109.0,109.2,109.9,118.1,119.9,120.3,120.7,120.9,123.2,123.3,123.4$, $123.7,125.7,126.6,128.0,128.2$, 129.3, 130.6, 131.5, 134.5, 138.2, 138.4, 140.8, 141.8, 151.4, 151.9, 152.1, 154.4, 159.1, 160.3, 160.9. Anal. Calcd. for $\mathrm{C}_{36} \mathrm{H}_{28} \mathrm{BrN}_{5} \mathrm{O}_{2}: \mathrm{C}, 67.29 ; \mathrm{H}, 4.39 ; \mathrm{N}, 10.90$. Found: C, 67.22; H, 4.35; N, 10.80 .

5-(9- Ethyl-9H- carbazol- 3- yl)- 1,3- dimethyl- 7-\{ 4-[( 3,4- dimethoxy-benzylidene)-amino]-phenyl $\}$ -1H-pyrido[2,3-d]-pyrimidine-2,4-dione (4i)

It was recrystallized from diethylether to give as yellow crystals. Yield $75.9 \%$, m.p. $259.6^{\circ} \mathrm{C}$; $\mathrm{IR}(\mathrm{KBr}$, v, $\left.\mathrm{cm}^{-1}\right): 1656.9\left(\mathrm{R}_{2} \mathrm{C}=\mathrm{N}-\mathrm{R}\right), 3049.5(\mathrm{C}=\mathrm{C}$, aromatic $), 1701.2(\mathrm{C}=\mathrm{O}), 2933.7\left(\mathrm{O}_{-} \mathrm{CH}_{3}\right) ;{ }^{1} \mathrm{HNMR}(300$ $\left.\mathrm{MHz}, \mathrm{CDCl}_{3}, \delta, \mathrm{ppm}\right): 9.03(1 \mathrm{H}, \mathrm{s},=\mathrm{CH}), 8.58(1 \mathrm{H}, \mathrm{s},=\mathrm{CH}), 8.37-8.39(1 \mathrm{H}, \mathrm{d},=\mathrm{CH}), 8.27-8.29(1 \mathrm{H}$, $\mathrm{d},=\mathrm{CH}), 7.16-7.71(12 \mathrm{H}, \mathrm{m},-\mathrm{Ar}-\mathrm{H}), 4.42-4.46\left(2 \mathrm{H}, \mathrm{q},-\mathrm{CH}_{2}\right), 3.86\left(6 \mathrm{H}, \mathrm{s},-\mathrm{OCH}_{3}\right), 3.71\left(3 \mathrm{H}, \mathrm{s},-\mathrm{CH}_{3}\right)$, $3.09\left(3 \mathrm{H}, \mathrm{s},-\mathrm{CH}_{3}\right), 1.32-1.36\left(3 \mathrm{H}, \mathrm{t},-\mathrm{CH}_{3}\right) .{ }^{13} \mathrm{CNMR}\left(300 \mathrm{MHz}, \mathrm{CDCl}_{3}, \delta, \mathrm{ppm}\right): 14.4,28.6,30.4,37.9$, 56.1, 56.5, 105.4, 109.7, 109.8, 109.9, 110.1, 111.9, 113.3, 117.6, 117.7, 119.9 (2C), 120.5, 120.7, 121.5, $123.1,123.2,124.9,125.8,125.9,126.8,128.4,130.1,130.2,130.3,140.8,141.6,149.7,149.8,154.8$, 
RASĀYAN J. Chem.

Vol. 11 | No. 3 |1286 - 1293 | July - September | 2018

158.8, 160.5, 160.8. Anal. Calcd. for $\mathrm{C}_{38} \mathrm{H}_{33} \mathrm{~N}_{5} \mathrm{O}_{4}: \mathrm{C}, 73.18 ; \mathrm{H}, 5.33 ; \mathrm{N}, 11.23$. Found: C, 73.16; H, $5.29 ; \mathrm{N}, 11.11$.

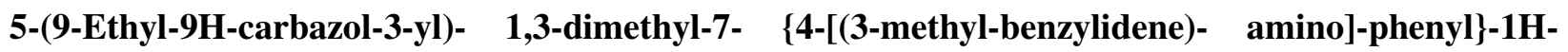 pyrido[2,3-d]-pyrimidine-2,4-dione $(4 \mathbf{j})$}

It was obtained after recrystallization from diethylether to give as yellow crystals. Yield $79.1 \%$, m.p. $226.6^{\circ} \mathrm{C}$; IR $\left(\mathrm{KBr}, v, \mathrm{~cm}^{-1}\right): 1658.8\left(\mathrm{R}_{2} \mathrm{C}=\mathrm{N}-\mathrm{R}\right), \quad 3049.5(\mathrm{C}=\mathrm{C}$, aromatic $), 1701.2(\mathrm{C}=\mathrm{O}), 2960.7$ $\left(-\mathrm{CH}_{3}\right) ;{ }^{1} \mathrm{HNMR}\left(300 \mathrm{MHz}, \mathrm{CDCl}_{3}, \delta, \mathrm{ppm}\right): 9.09(1 \mathrm{H}, \mathrm{s},=\mathrm{CH}), 8.67(1 \mathrm{H}, \mathrm{s},=\mathrm{CH}), 8.42-8.45(1 \mathrm{H}, \mathrm{d}$, $=\mathrm{CH}), 8.29-8.33(1 \mathrm{H}, \mathrm{d},=\mathrm{CH}), 7.18-7.82(13 \mathrm{H}, \mathrm{m},-\mathrm{Ar}-\mathrm{H}), 4.44-4.50\left(2 \mathrm{H}, \mathrm{q},-\mathrm{CH}_{2}\right), 3.75\left(3 \mathrm{H}, \mathrm{s},-\mathrm{CH}_{3}\right)$, $3.15\left(3 \mathrm{H}, \mathrm{s},-\mathrm{CH}_{3}\right), 2.41\left(3 \mathrm{H}, \mathrm{s},-\mathrm{CH}_{3}\right), 1.32-1.36\left(3 \mathrm{H}, \mathrm{t},-\mathrm{CH}_{3}\right) .{ }^{13} \mathrm{CNMR}\left(300 \mathrm{MHz}, \mathrm{CDCl}_{3}, \delta, \mathrm{ppm}\right)$ : $14.5,21.6,28.6,30.4,37.9,105.6,109.9,110.0,110.1,117.7,117.8,120.0,120.1,120.8,120.9,121.5$, $121.6,123.2,123.3,126.8,126.9,128.1,129.4,129.6,129.7,130.2,130.4,135.9,136.7,138.8,141.7$, 151.7, 151.9, 154.1, 159.4, 160.4, 161.4. Anal. Calcd. for $\mathrm{C}_{37} \mathrm{H}_{31} \mathrm{~N}_{5} \mathrm{O}_{2}: \mathrm{C}, 76.93 ; \mathrm{H}, 5.41 ; \mathrm{N}, 12.12$. Found: C, 76.89; H, 5.39; N, 12.10 .

5-(9- Ethyl- 9H- carbazol-3-yl)- 1,3-dimethyl-7-\{4-[(2-nitro-benzylidene)-amino]- phenyl $\}-1 H-$ pyrido[2,3-d]-pyrimidine-2,4-dione (4k)

It was recrystallized from diethylether to give as brown crystals. Yield $87.2 \%$, m.p. $285.9^{\circ} \mathrm{C}$; $\mathrm{IR}(\mathrm{KBr}$, v, $\left.\mathrm{cm}^{-1}\right): \quad 1656.9\left(\mathrm{R}_{2} \mathrm{C}=\mathrm{N}-\mathrm{R}\right), \quad 3047.5(\mathrm{C}=\mathrm{C}$, aromatic $), 1703.1 \quad(\mathrm{C}=\mathrm{O}), 1583.6\left(\mathrm{NO}_{2}\right) ;{ }^{1} \mathrm{HNMR}(300$ $\left.\mathrm{MHz}, \mathrm{CDCl}_{3}, \delta, \mathrm{ppm}\right): 9.07(1 \mathrm{H}, \mathrm{s},=\mathrm{CH}), 8.88(1 \mathrm{H}, \mathrm{s},=\mathrm{CH}), 8.34-8.36(1 \mathrm{H}, \mathrm{d},=\mathrm{CH}), 8.18-8.21(1 \mathrm{H}$, $\mathrm{d},=\mathrm{CH}), 8.09-8.12(1 \mathrm{H}, \mathrm{d},=\mathrm{CH}), 7.62(1 \mathrm{H}, \mathrm{s},=\mathrm{CH}), 7.26-7.80(11 \mathrm{H}, \mathrm{m},-\mathrm{Ar}-\mathrm{H}), 4.39-4.46(2 \mathrm{H}, \mathrm{q}$, - $\left.\mathrm{CH}_{2}\right), 3.96\left(3 \mathrm{H}, \mathrm{s},-\mathrm{CH}_{3}\right), 3.42\left(3 \mathrm{H}, \mathrm{s},-\mathrm{CH}_{3}\right), 1.46-1.51\left(3 \mathrm{H}, \mathrm{t},-\mathrm{CH}_{3}\right) .{ }^{13} \mathrm{CNMR}\left(300 \mathrm{MHz}, \mathrm{CDCl}_{3}, \delta\right.$, ppm): 14.1, 28.7, 30.5, 38.1, 105.7, 109.0, 109.2, 118.1, 119.9, 120.3, 120.9, 121.1, 123.3, 123.7, 124.8, $125.7,126.6,128.2,129.4,129.8,130.0,130.1,131.4,131.5,133.8,133.9,138.8,140.8,141.9,149.5$, 151.9, 152.1, 154.3, 160.4, 160.9, 161.0. Anal. Calcd. for $\mathrm{C}_{36} \mathrm{H}_{28} \mathrm{~N}_{6} \mathrm{O}_{4}: \mathrm{C}, 71.04 ; \mathrm{H}, 4.64 ; \mathrm{N}, 13.81$. Found: C, 70.96; H, 4.56; N, 13.73 .

5-(9-Ethyl-9H-carbazol-3-yl)-1,3-dimethyl-7-\{4-[(3,4-dichloro-benzylidene)-amino]-phenyl $\} \quad$-1Hpyrido [2,3-d]-pyrimidine-2,4-dione (4I)

It was recrystallized from diethylether to give as brown crystals. Yield $86.4 \%$, m.p. $228.3^{\circ} \mathrm{C}$; IR $(\mathrm{KBr}$, v, $\left.\mathrm{cm}^{-1}\right): 1658.8\left(\mathrm{R}_{2} \mathrm{C}=\mathrm{N}-\mathrm{R}\right), 3047.5(\mathrm{C}=\mathrm{C}$, aromatic $), 1699.3(\mathrm{C}=\mathrm{O}), 746.5(\mathrm{C}-\mathrm{Cl})$;

${ }^{1} \mathrm{HNMR}\left(300 \mathrm{MHz}, \mathrm{CDCl}_{3}, \delta, \mathrm{ppm}\right): 8.88(1 \mathrm{H}, \mathrm{s},=\mathrm{CH}), 8.49(1 \mathrm{H}, \mathrm{s},=\mathrm{CH}), 8.34-8.37(1 \mathrm{H}, \mathrm{d},=\mathrm{CH})$, 8.18-8.21 $(1 \mathrm{H}, \mathrm{d},=\mathrm{CH}), 8.06(1 \mathrm{H}, \mathrm{s},=\mathrm{CH}), 7.62(1 \mathrm{H}, \mathrm{s},=\mathrm{CH}), 7.26-7.77(10 \mathrm{H}, \mathrm{m},-\mathrm{Ar}-\mathrm{H}), 4.39-4.46$ $\left(2 \mathrm{H}, \mathrm{q},-\mathrm{CH}_{2}\right), 3.96\left(3 \mathrm{H}, \mathrm{s},-\mathrm{CH}_{3}\right), 3.42\left(3 \mathrm{H}, \mathrm{s},-\mathrm{CH}_{3}\right), 1.46-1.51\left(3 \mathrm{H}, \mathrm{t},-\mathrm{CH}_{3}\right) .{ }^{13} \mathrm{CNMR}(300 \mathrm{MHz}$, $\left.\mathrm{CDCl}_{3}, \delta, \mathrm{ppm}\right): 14.1,28.7,30.5,38.1,105.7,109.0,109.2,109.9,118.1,119.9,120.3,120.7,120.9$, $121.0,123.3,123.7,125.7,126.5,126.6,128.2$, 129.4, 130.4, 131.1, 133.5, 135.7, 136.3, 138.4, 140.8, 141.9, 151.1, 151.9, 152.1, 154.4, 160.3, 160.6, 160.9. Anal. Calcd. for $\mathrm{C}_{36} \mathrm{H}_{27} \mathrm{Cl}_{2} \mathrm{~N}_{5} \mathrm{O}_{2}$ : C, 68.36; $\mathrm{H}$, 4.30; N, 11.07. Found: C, 68.33; H, 4.29; N, 11.04 .

5-(9- Ethyl- 9H- carbazol-3-yl)- 1,3-dimethyl-7-\{4-[(2-bromo-benzylidene)-amino]- phenyl\}-1Hpyrido[2,3-d]-pyrimidine-2,4-dione (4m)

It was obtained after recrystallization from diethylether to give as orange crystals. Yield $72.6 \%$, m.p. $247.8^{\circ} \mathrm{C} ;{ }^{1} \mathrm{HNMR}\left(300 \mathrm{MHz}, \mathrm{CDCl}_{3}, \delta, \mathrm{ppm}\right): 9.15(1 \mathrm{H}, \mathrm{s},=\mathrm{CH}), 8.89(1 \mathrm{H}, \mathrm{s},=\mathrm{CH}), 8.46-8.49(1 \mathrm{H}, \mathrm{d}$, $=\mathrm{CH}), 8.32-8.35(1 \mathrm{H}, \mathrm{d},=\mathrm{CH}), 8.19-8.21(1 \mathrm{H}, \mathrm{d},=\mathrm{CH}), 7.84(1 \mathrm{H}, \mathrm{s},=\mathrm{CH}), 7.50-7.79(9 \mathrm{H}, \mathrm{m},-\mathrm{Ar}-\mathrm{H})$, 7.36-7.38 $(1 \mathrm{H}, \mathrm{t},=\mathrm{CH}), 7.26-7.28(1 \mathrm{H}, \mathrm{t},=\mathrm{CH}), 4.46-4.51\left(2 \mathrm{H}, \mathrm{q},-\mathrm{CH}_{2}\right), 3.79\left(3 \mathrm{H}, \mathrm{s},-\mathrm{CH}_{3}\right), 3.19(3 \mathrm{H}, \mathrm{s}$, $\left.-\mathrm{CH}_{3}\right), 1.33-1.38\left(3 \mathrm{H}, \mathrm{t},-\mathrm{CH}_{3}\right) .{ }^{13} \mathrm{CNMR}\left(300 \mathrm{MHz}, \mathrm{CDCl}_{3}, \delta, \mathrm{ppm}\right): 13.9,28.4,30.2,37.8,105.4,108.7$, $108.9,117.8,119.6,119.9,120.6,120.7,120.8,121.2$, 122.9, 123.3, 125.4, 125.5, 126.3, 126.6, 127.7, 127.9, 129.0, 129.1, 132.5, 133.2, 134.5, 137.9, 140.5, 141.5, 151.3, 151.6, 154.1, 159.6, 159.9, 160.7. Anal. Calcd. for $\mathrm{C}_{36} \mathrm{H}_{28} \mathrm{BrN}_{5} \mathrm{O}_{2}$ : C, 67.29; H, 4.39; N, 10.90. Found: C, 67.22; H, 4.36; N, 10.86 .

5-(9-Ethyl- 9H- carbazol-3-yl)-1,3-dimethyl-7-\{4-[(2,5-dimethoxy-benzylidene)-amino]-phenyl\}-1Hpyrido[2,3-d]-pyrimidine-2,4-dione (4n)

It was recrystallized from diethylether to give as orange crystals. Yield $84.3 \%$, m.p. $276.1^{\circ} \mathrm{C} ;{ }^{1} \mathrm{HNMR}$ (300 MHz, $\left.\mathrm{CDCl}_{3}, \delta, \mathrm{ppm}\right): 9.16(1 \mathrm{H}, \mathrm{s},=\mathrm{CH}), 8.91(1 \mathrm{H}, \mathrm{s},=\mathrm{CH}), 8.48-8.50(1 \mathrm{H}, \mathrm{d},=\mathrm{CH}), 8.33-8.36$ 
$(1 \mathrm{H}, \mathrm{d},=\mathrm{CH}), 7.85(1 \mathrm{H}, \mathrm{s},=\mathrm{CH}), 7.15-7.77(11 \mathrm{H}, \mathrm{m},-\mathrm{Ar}-\mathrm{H}), 4.48-4.54\left(2 \mathrm{H}, \mathrm{q},-\mathrm{CH}_{2}\right), 3.88(6 \mathrm{H}, \mathrm{s}$, $\left.=\mathrm{CH}_{3}\right), 3.81\left(3 \mathrm{H}, \mathrm{s},-\mathrm{CH}_{3}\right), 3.22\left(3 \mathrm{H}, \mathrm{s},-\mathrm{CH}_{3}\right), 1.33-1.38\left(3 \mathrm{H}, \mathrm{t},-\mathrm{CH}_{3}\right) .{ }^{13} \mathrm{CNMR}\left(300 \mathrm{MHz}, \mathrm{CDCl}_{3}, \delta\right.$, ppm): 14.1, 28.7, 30.5, 38.1, 56.2, 56.5, 105.7, 109.0, 109.2, 110.5, 113.1, 118.2, 119.9, 120.2, 120.3, $120.9,121.0,123.3,123.7,125.3,125.7,126.6,128.3,128.5,129.2,131.5,137.4,140.8,141.8,151.9$, 152.1, 152.8, 153.9, 154.6, 154.7, 157.2, 160.3, 161.0. Anal. Calcd. for $\mathrm{C}_{38} \mathrm{H}_{33} \mathrm{~N}_{5} \mathrm{O}_{4}: \mathrm{C}, 73.18 ; \mathrm{H}, 5.33$; N, 11.23. Found: C, 73.13; H, 5.29; N, 11.17.

\section{RESULTS AND DISCUSSION}

The synthesis of pyridopyrimidine derivatives of carbazole containing nitro, amino and imino groups have resulted in a good yield. The nitro chalcone derivative (1) was prepared in a good yield according to the following procedures mentioned in literature ${ }^{45}$ and it was converted to the pyridopyrimidine derivatives (2) using 6-amino-1,3-dimethyluracil with $\mathrm{NaOH}$ as a base catalyst in ethanol. In anhydrous tetrahydrofuran using nickel (II) chloride, the nitro compound was reducted to the compound bearing amino group (3). The amino pyridopyrimidine compound reacted with various benzaldehyde derivatives form series of different compounds (4a-n) in ethanol in high yield.

The structures of the prepared compounds (4a-n) were deduced from their elemental analysis data, and from their IR, ${ }^{1} \mathrm{H}-\mathrm{NMR}$ and ${ }^{13} \mathrm{C}-\mathrm{NMR}$ spectra.The IR spectra of all the compounds showed a characteristic mode at $1660.7 \mathrm{~cm}^{-1}$ which may be resulted from vibrations of group $\mathrm{R}_{2} \mathrm{C}=\mathrm{N}-\mathrm{R}$. The characteristic modes at $3050 \mathrm{~cm}^{-1}$ and $1703.1 \mathrm{~cm}^{-1}$ resulted from aromatic stretching frequencies and a corresponding carbonyl group. The absorption band at $1145.7 \mathrm{~cm}^{-1}$ indicated the presence of C-F group for the compound $\mathbf{4 c}$. The compounds $\mathbf{4 b}$ and $\mathbf{4 k}$ showed a characteristic mode at around $1583 \mathrm{~cm}^{-1}$ which may be resulted from vibrations of $\mathrm{NO}_{2}$ group. The $\mathrm{C}-\mathrm{Cl}$ bond stretching frequencies appeared around $746 \mathrm{~cm}^{-1}$ for $\mathbf{4 c}$ and $4 \mathbf{l}$. In the ${ }^{1} \mathrm{H}-\mathrm{NMR}$ spectrum of the compounds, aromatic protons were appeared around $\delta 7.20-9.0 \mathrm{ppm}$. The spectrum also displayed a singlet at $\delta 8.59 \mathrm{ppm}(\mathrm{s}, 1 \mathrm{H})$ assigned for group $(-\mathrm{N}=\mathrm{CH})$, absorption signals around $\delta 3.43 \mathrm{ppm}$ and $\delta 3.97 \mathrm{ppm}$ correspond to the six protons of the two groups $\mathrm{N}-\mathrm{CH}_{3}$, a quartet between $\delta 4.39-4.47 \mathrm{ppm}(\mathrm{q}, 2 \mathrm{H})$ assigned for $\mathrm{CH}_{2}$ protons and a triplet in the range $\delta 1.47-1.51 \mathrm{ppm}(\mathrm{t}, 3 \mathrm{H})$ correspond to $\mathrm{CH}_{3}$ protons. Singlet for the protons of the methoxy group is observed in the area of around 3,92 ppm for the compounds $4 \mathbf{d d}, \mathbf{4 e}, \mathbf{4 i}$ and $\mathbf{4 n}$. From the ${ }^{13} \mathrm{C}$ NMR spectra, two signals from the carbonyl groups present in the pyrimidine ring were observed respectively around 160 and $151 \mathrm{ppm}$. The characteristic signals for $\mathrm{C}=\mathrm{N}, \mathrm{C}-\mathrm{NO}_{2}, \mathrm{OCH}_{3}, \mathrm{CH}_{2}$ and $\mathrm{CH}_{3}$ groups are respectively around 160,140, 55, 38 and $14 \mathrm{ppm}$ respectively. Also the spectrum displayed two signals at 30.5 and $28.7 \mathrm{ppm}$ for the carbon atoms of $\mathrm{N}_{-} \mathrm{CH}_{3}$ groups. The obtained structures are confirmed based on IR, ${ }^{1} \mathrm{H}$ NMR and ${ }^{13} \mathrm{C}$ NMR spectra and the results obtained from the elemental analysis have been consistent with the composition of the newly synthesized compounds.

\section{CONCLUSION}

The new Schiff bases which have carbazole bearing pyridopyrimidine group have been reported as one of the most important reactions in organic and medicinal chemistry. The pyridopyrimidine motif is widely used in many organic compounds, and drug activity was related to biological activities. The synthesis procedures were performed under mild reaction conditions, in the absence of the catalyst providing the corresponding products in a good yield.

\section{ACKNOWLEDGMENT}

We thank the Research Fund of the Sakarya University for financial support. Project Number: 2016-0204-018.

\section{REFERENCES}

1. A.R. Nixha, H. Demirhan and M.Arslan, Rasayan J. Chem., 10(4), 1374 (2017), DOI:10.7324 /RJC.2017.1041883

2. A. Solankee, G. Patel and S. Solankee, Rasayan J. Chem., 1(3), 591(2008).

3. M. Salahuddin, S. Singh and S. M.Shantakumar, Rasayan J. Chem., 2(1), 167(2009).

4. M. A. El-Remaily, O. M. El Hady, H. S. Zaid and E. M. M. El-Raheem, J. of Heterocyclic Chem., 53(4), 1304, (2016), DOI:10.1002/jhet.2420 
RASĀYAN J. Chem.

Vol. 11 | No. 3 |1286 - 1293 | July - September | 2018

5. I.S. Zorkun, S. Sarac, S. Celebi and K. Erol, Bioorg. Med. Chem., 14(24), 8582(2006), DOI: $10.1016 / j . b m c .2006 .08 .031$

6. A. Nagaraj and C. Sanjeeva Reddy, J. Iran. Chem. Soc., 5, 262 (2008).

7. R.B. Patel and K.H. Chikhalia, Indian J. Chem., 45B, 1871(2006).

8. A.R. Saundane, K. Vijaykumar, A. V. Vaijinath and P. Walmik, Med Chem Res., 22, 806(2013), DOI: $10.1155 / 2013 / 530135$

9. A. Anu, S. Kumkum, K.P. Puri and M.S.C. Prem, Bioorg. Med. Chem. Lett., 15(7), 1881(2005).

10. K. Rana, B. Kaur and B. Kumar, Indian J. Chem., 13, 1553(2004).

11. V.R. Chilkale, P.R. Bhole, B.P. Khadekar and P.K. Bhusari, European. J. Med. Chem., 44, 3645 (2009), DOI: 10.1016/j.ejmech.2009.02.021

12. H. Kuday, F. Sonmez, C. Bilen, E. Yavuz, N. Gençer and M. Kucukislamoglu, Biomed. Res. Int., Article ID 594879 (2014), DOI: 10.1155/2014/594879

13. S.M. Sondhi, N. Singh and M. Johar, Bioorg. Med. Chem., 13(22), 6158 (2005).

14. R.B. Dravyakar, P.D. Kawade and P.K. Bhusari, Indian. J. Heterocycl. Chem., 16(3), 301(2007).

15. M.A. Azam, B.R.P. Kumar, S. Shalini, B. Suresh, T.K. Reddy and C.D. Reddy, Indian. J.Pharm. Sci., 70(5), 672 (2008), DOI: 10.4103/0250-474X.45416

16. A. M. Mohamed, W.A. El-Sayed, M.A. Alsharari, H. R. M. Al-Qalawi and M. O. Germoush, Arch. Pharm. Res., 36, 1055 (2013), DOI: 10.1007/s12272-013-0163-X

17. C. Shilpa, S. Dipak, S. Vimukta and D. Arti, International Journal of Pharmaceutical Sciences Review and Research, 15(1),15(2012).

18. S.L. Buchwald, C. Mauger, G. Mignani and U. Scholz, Adv. Synth. Catal., 348, 23 (2006).

19. S. Cacchi and G. Fabrizi, Chem. Rev., 105, 2873 (2005), DOI: 10.1021/cr040639b

20. L. Djakovitch, V. Dufaud and R. Zaidi, Adv. Synth. Catal., 348,715(2006), DOI: 10.1002/adsc.200505283

21. V. Balraju and J. Iqbal, J. Org. Chem., 71, 8954 (2006), DOI: 10.1021/jo061366i

22. A. Zanardi, J.A. Mata and E. Peris, Chem.-Eur. J., 16,10502(2010), DOI: 10.1002/chem.201000801

23. M.S. Kwon, S.J. Kim, S.H. Park, W. Bosco, R.K. Chidrala and J.W. Park, J. Org. Chem., 74, 2877 (2009).

24. T. Ishida, N. Kawakita, T. Akita and M. Haruta, Gold Bulletin, 42, 267 (2009).

25. N. Uematsu, A. Fujii, S. Hashiguchi, T. Ikariya and R. Noyori, J. Am. Chem. Soc., 118, 4916 (1996), DOI: $10.1021 / \mathrm{ja} 960364 \mathrm{k}$

26. J.P. Adams, J. Chem. Soc., Perkin Trans., 1(2), 125 (2000).

27. A. Cordova, 'Acc. Chem. Res., 37, 102 (2004), DOI: 10.1021/ar0302311

28. W.J. Drury III, D. Ferraris, C. Cox, B. Young and T. Lectka, J. Am. Chem. Soc., 120, 11006(1998), DOI: $10.1021 / \mathrm{ja} 982257 \mathrm{r}$

29. M. Shi and Y.M. Xu, Angew. Chem. Int. Ed., 41, 4507(2002), DOI: 10.1002/15213773(20021202)41:23<4507::AID-ANIE4507>3.0.CO;2-I

30. R. Bloch, Chem. Rev., 98, 1407 (1998), DOI: 10.1021/cr940474e

31. F. Bellezza, A. Cipiciani, U. Costantino, F. Fringuelli, M. Orru, O. Piermatti and F. Pizzo, 'Catal. Today, 152, 61 (2010).

32. A. Hoepping, K.M. Johnson, C. George, J. Flippen-Anderson and A.P. Kozikowski, J. Med. Chem., 43, 2064 (2000).

33. C. Swithenbank, P.J. McNulty and K.L. Viste, J. Agric. Food Chem., 19, 417 (1971).

34. E.M. Kosower, A.E. Radkowsky, A.H. Fairlamb, S.L. Croft and R.A. Nea, Eur. J. Med. Chem., 30, 659 (1995), DOI: 10.1016/0223-5234(96)88283-3

35. M. Keller, A.S.S. Sido, P. Pale and J. Sommer, Chem.-Eur. J., 15, 2810 (2009), DOI: 10.1002/chem.200802191

36. R.W. Layer, Chem. Rev., 63(5), 489 (1963), DOI: 10.1021/cr60225a003

37. B. Das, B. Ravikanth, K. Laxminarayana and B.V. Rao, J. Mol. Catal. A: Chem., 253, 92 (2006).

38. F.Z. Su, S.C. Mathew, L. Mohlmann, M. Antonietti, X.C. Wang and S. Blechert, Angew. Chem. Int. Ed., 50, 657 (2011), DOI:10.1002/anie.201004365

39. A. Grirrane, A. Corma and H. Garcia, J. Catal., 264, 138 (2009).

SCHIFF BASE SUBSTITUTED CARBAZOLE 
40. B.L. Zhu and R.J. Angelici, Chem. Commun., 21, 2157(2007).

41. M.H. So, Y.G. Liu, C.M. Ho and C.M. Che, Chem.-Asian J., 4, 1551(2009), DOI: 10.1002/asia.200900261

42. H. Sun, F.Z. Su, J. Ni, Y. Cao, H.Y. He, H.Y and K.N. Fan, Angew. Chem., Int. Ed., 48, 4390 (2009), DOI:10.1002/anie.200900802

43. S. Kegnæs, J. Mielby, U.V. Mentzel, C.H. Christensen and A. Riisager, Green Chem., 12, 1437 (2010).

44. B. Gnanaprakasam, J. Zhang and D. Milstein, Angew. Chem., Int. Ed., 49, 1468(2010), DOI: 10.1002/anie.200907018

45. A.R. Nixha, M. Arslan, Y. Atalay, N. Gencer, A. Ergun and O. Arslan, Journal Of Enzyme Inhibition and Medicinal Chemistry, 28(4), 808(2013), DOI: 10.3109/14756366.2012.688040

[RJC-4014/2018] 